\section{Evaluación de virus papiloma humano de alto y bajo riesgo oncogénico en la cavidad bucal de pacientes VIH positivos}

\section{Artículo Original}

Maira Ávila ${ }^{1, a}$, Victor Briceño 2,a Andreína Fernandes ${ }^{1, b}$ Maglynert Montero ${ }^{1, c}$, María Correnti ${ }^{1, b}$, Williams Carrasco $^{3, d}$

${ }^{1}$ Universidad Central de Venezuela, Instituto de Investigaciones Odontológicas "Raúl Vincentelli", Facultad de Odontología, Caracas, Venezuela.

${ }^{2}$ Laboratorio de Genética Molecular, Instituto de Oncología y Hematología, MPPPS, Caracas, Venezuela. ${ }^{3}$ Universidad Central de Venezuela, Facultad de Odontología, Centro de Atención a Pacientes con Enfermedades Infecciosas, Caracas, Venezuela.

a Licenciado(a) en Biología.

${ }^{\mathrm{b}}$ Doctora en Ciencias Biológicas.

c Odontólogo

${ }^{d}$ Doctor en Odontología.

\section{Evaluation of human papillomavirus of high and low oncogenic risk in the oral cavity of HIV positive patients}

\begin{abstract}
Resumen
Objetivo. Realizar la detección y tipificación de virus papiloma humano (VPH) en la cavidad bucal de un grupo de pacientes VIH positivos, atendidos en el Centro de Atención de Pacientes con Enfermedades Infectocontagiosas (CAPEI) de la Facultad de Odontología-Universidad Central de Venezuela. Métodos. Se evaluaron 31 hisopados bucales de pacientes VIH positivos para la infección por VPH, mediante el Sistema de Hibridación Reversa de INNO-LiPA que detecta 28 genotipos de VPH de alto y bajo riesgo oncogénico. Resultados. El 61,0\% de las muestras evaluadas presentó infección por VPH. El genotipo 6 fue el más frecuente (73,68\%), seguido de los genotipos 18, 11 y 16 con $63,16 \%, 37,0 \%$ y $32,0 \%$ respectivamente. El $74,0 \%$ de las muestras positivas para VPH presentaron infecciones múltiples, siendo más frecuente la coinfección mixta (35,70\%) con los genotipos de VPH-6/18 de bajo y alto riesgo oncogénico, 21,40\% con los genotipos $6 / 11 / 18$ y $6 / 11$, cada una. Seguido de $14,30 \%$ de las muestras que presentaron infección con VPH-6/11/16/18 y 7,10\% con los genotipos 11/16 de bajo y alto riesgo oncogénico. Conclusiones. La alta frecuencia de infección con VPH de alto riesgo oncogénico y la presencia de múltiples genotipos observada en la cavidad bucal de individuos VIH positivos, que no presentaron lesiones compatibles con esta infección en el examen extra e intrabucal, indica que los métodos moleculares de diagnóstico son importantes en la detección de infecciones subclínicas y latentes, lo que puede permitir un mejor seguimiento y manejo más oportuno de estos pacientes con mayor riesgo de desarrollar neoplasias malignas en la cavidad bucal.
\end{abstract}

Palabras clave: Infección por VPH; Infecciones por VIH; Cavidad bucal (fuente: DeCS BIREME).

\begin{abstract}
Objective. Perform human papilomavirus (HPV) detection and typing in the oral cavity in a group of HIV positive patients, treated at the Center for Care of Patients with Infectious Diseases (CAPEI) of the Faculty of Dentistry-Central University of Venezuela. Methods. Thirty-one oral swabs from HIV-positive patients were evaluated to detect HPV infection using the INNO-LiPA Reverse Hybridization System that detects 28
\end{abstract}

\section{Correspondencia:}

Maira Ávila: avimaira@gmail.com

Av. Los Ilustres, Ciudad Universitaria, Edif. Facultad de Odontología, piso 9, Instituto de Investigaciones Odontológicas "Raúl Vincentelli", Los Chaguaramos, UCV, Caracas- Venezuela. Código postal: 1051. ORCID: 0000-0002-1624-6633

\section{Coautores:}

Victor Briceño: victor752_2@hotmail.com. ORCID: 0000-0002-8249-7756

Andreína Fernandes: andreinafernandes@yahoo.es ORCID: 0000-0003-0589-2696

Maglynert Montero: maglymontero44@gmail.com ORCID: 0000-0003-0829-400X

María Correnti. mcorrentip@yahoo.com

ORCID: 0000-0001-7394-1352

Williams Carrasco: wcarrasco12@hotmail.com ORCID: 0000-0001-7732-3631

\section{Editor:}

Juan Carlos Cuevas-González

Universidad Autónoma de Ciudad Juárez, México.

Conflicto de intereses: los autores declaran no tener conflictos de interés.

Fuente de financiamiento: proyecto Estratégico FONACIT No. 2011000413.

Recibido: 11/03/20

Aceptado: 08/04/20

Publicado: 04/08/20 
HPV genotypes of high and low oncogenic risk. Results. The $61.0 \%$ of the evaluated samples had an HPV infection. The low risk genotype 6 was the most frequent $(73.68 \%)$, followed by genotypes 18,11 and 16 with $63.16 \%, 37.0 \%$, and $32.0 \%$, respectively. Seventy-four percent of HPV positive samples had multiple infections, being the more frequent the mixed coinfection (35.70\%), with HPV genotypes 6/18 of low and high oncogenic risk, $21.40 \%$ with genotypes $11 / 6 / 18$ and $6 / 11$, each one, followed by $14.30 \%$ of the samples presented infection with HPV-6/11/16/18 and 7.10\% with the 11/16 genotypes of low and high oncogenic risk. Conclusions. The high frequency of infection with high oncogenic risk HPV types and the presence of multiple genotypes observed in the oral cavity of HIV positive patients, who did not present lesions compatible with this infection in the extra and intraoral examination, indicates that molecular diagnostic methods are important in the detection of subclinical and latent infections, which may allow better follow-up and more timely management of these patients with a higher risk of developing malignant lesions in the oral cavity.

Keywords: HPV infection; HIV infections; Oral cavity (source: MeSH NLM).

\section{Introducción}

El virus de inmunodeficiencia humana (VIH), es el agente etiológico del síndrome de inmunodeficiencia adquirida (SIDA), caracterizado por una alteración del sistema inmunitario con una progresiva disminución de los linfocitos T CD4, que origina una profunda inmunosupresión con diversas particularidades clínicas, incluidas las infecciones oportunistas, las neoplasias malignas y la degeneración del sistema nervioso central ${ }^{1-4}$.

Hoy en día se considera que las manifestaciones bucales de la infección por VIH son un indicador de inmunosupresión temprana y de progresión de la enfermedad, debido que entre el 70- 90\% de las personas infectadas con este virus presentan manifestaciones bucales en algún estadio, y en ocasiones estas son el primer signo de la enfermedad ${ }^{5-9}$. Diversos estudios han identificado las lesiones bucales como marcadores del deterioro de la respuesta inmunitaria; independientemente del recuento de linfocitos T CD4 y del progreso del SIDA en los pacientes infectados ${ }^{10,11}$. Un recuento de linfocitos $T$ CD 4 menor de 200 células $/ \mathrm{mm}^{3}$, una carga viral mayor de 30000 copias $/ \mathrm{mL}$, la presencia de xerostomía, una pobre higiene oral y el hábito de fumar son factores que predisponen a la expresión de lesiones en la cavidad bucal en estos pacientes ${ }^{12}$.

Es importante mencionar, que en los últimos años con la introducción de la Terapia Antirretroviral Altamente Activa (HAART) se ha modificado la incidencia de infecciones oportunistas en la cavidad bucal y se ha provisto de una alternativa que ha permitido prolongar y mejorar la calidad de vida de los pacientes con VIH y llevar esta enfermedad infecciosa a un estado de cronicidad ${ }^{3}$, sin embargo se ha observado un marcado incremento de la incidencia de verrugas bucales, así como de papilomas y condilomas asociados a la infección por virus papiloma humano (VPH) ${ }^{13-15}$.

Los VPH son altamente específicos de especie y tejidos, muestran un tropismo exclusivo por las células epiteliales de las mucosas y de la piel, y pueden producir una infección clínica, subclínica o latente. En estas tres presentaciones las manifestaciones clínica pueden ocurrir y recurrir durante la infección. Estos virus son miembros de la familia Papillomaviridae, presentan una cápside icosahédrica proteica de 72 capsómeros, que encierra una molécula de $\mathrm{ADN}$ de doble cadena circular, cerrada y superenrollada, de $8 \mathrm{~Kb}^{16}$.

Basados en su asociación con el cáncer cervical y las lesiones precursoras, estos virus se han agrupado en genotipos de alto y bajo riesgo oncogénico. Los genotipos $6,11,42,43$ y 44, están comúnmente asociados con el condiloma acuminado y displasias menores, razón por la cual se les ha denominado de "bajo riesgo"; mientras que los tipos 16, 18, 31, 33, 34, 35, 39, 45 entre otros, son considerados de "alto riesgo oncogénico" debido a su asociación con el desarrollo de la neoplasia intraepítelial cevical (NIC), carcinoma in situ, cáncer cervical y más recientemente con los cánceres de cabeza y cuello, específicamente los de orofaringe ${ }^{17}$.

En la cavidad bucal, los VPH son responsables de múltiples manifestaciones que pueden incluir lesiones benignas como el papiloma bucal, verruga vulgar bucal, condiloma acuminado bucal e hiperplasia epitelial multifocal, mientras que las lesiones premalignas o malignas incluyen la leucoplasia y el carcinoma escamocelular ${ }^{18}$. Es importante mencionar que diferentes estudios han mostrado un aumento en la incidencia de la infección de estos virus en cavidad bucal, debido a que el sexo oro-genital es una de las principales vías de contagio, lo que también permite que cada vez exista un mayor número de diagnósticos de VPH de alto riesgo (VPH-AR) oncogénico en cavidad bucal ${ }^{19}$.

En el caso específico de individuos VIH positivos, algunos estudios han reportado hasta un $56 \%$ de infección por VPH en la cavidad bucal, una proporción sustancialmente superior a la reportada en poblaciones no infectadas por el VIH ${ }^{20-22}$. En este sentido, una investigación realizada en 314 hombres VIH positivos reportó que el $24,8 \%$ de los mismos presentaba infección con VPH-AR oncogénico, mientras que en los individuos VIH negativos la infección con VPH-AR oncogénico solo estuvo presente en el 8,8 \%, siendo el genotipo más común el $16^{23}$. 
Otro estudio donde se evaluó la mucosa bucal de 52 individuos VIH positivos, para detectar la presencia de $\mathrm{VPH}$, reportó que el $87 \%$ de los mismos presentaban infección por VPH, de los cuales $23 \%$ tenían VPH-AR oncogénico y $40 \% \mathrm{VPH}-\mathrm{BR}$ oncogénico. Este estudio además reportó una relación significativa entre la detección de VPH y el aumento de la carga viral del VIH ${ }^{24}$.

Considerando que en pacientes VIH positivos la prevalencia de la infección con VPH-AR en la mucosa bucal es mayor que en la población general, y que además presentan mayor riesgo de desarrollar cánceres relacionados con estos virus, este trabajo tuvo como objetivo, establecer la frecuencia de la infección por VPH de alto y bajo riesgo oncogénico en la cavidad bucal de un grupo de pacientes VIH positivos.

\section{Métodos}

El presente trabajo correspondió a un estudio descriptivo, transversal, de tipo experimental.

Muestra de estudio. Se incluyeron 31 pacientes mayores de edad de ambos sexos ( 23 hombres y 8 mujeres), con diagnóstico confirmado de infección por VIH con registro de la carga viral y contaje de linfocitos T CD4 en la historia clínica, que acudieron al Centro de Atención a Personas con Enfermedades Infectocontagiosas (CAPEI), de la Facultad de Odontología de la Universidad Central de Venezuela (UCV), entre el período de enero a marzo de 2017.

Se excluyeron del estudio mujeres embarazadas, pacientes con diagnóstico de diabetes mellitus, pacientes que consumieran antidepresivos y pacientes con tratamiento de ortodoncia. Previo a la toma de muestra, los pacientes firmaron un consentimiento informado aprobado por el Comité de Bioética de la Facultad de Odontología de la UCV No. 031-2016.

Recolección de las muestras de hisopado bucal. Para la recolección de las muestras de hisopados bucales, un odontólogo realizó un examen intra y extrabucal, para verificar lesiones en tejidos blandos y duros. Posteriormente, con el hisopo de dacrón se realizó un barrido por la mucosa oral que incluyó: lengua, borde de lengua, piso de boca, paladar y carrillo. Seguidamente, el hisopo se colocó en un tubo con medio de transporte y se guardó a $-20^{\circ} \mathrm{C}$ hasta el momento de su procesamiento.

Detección y Tipificación de Virus papiloma humano. Previo a la detección y tipificación de VPH en las muestras de hisopados bucales se llevó a cabo el proceso de extracción y purificación del material genómico en cuatro pasos que incluyeron: preparación del lisado celular, unión, lavado y elución del ADN, mediante el estuche comercial Purelink Genomic DNA de Invitrogen.

Una vez extraído el material genómico se procedió a detectar y tipificar el VPH. Para ello se utilizó el Kit INNO-LiPA HPV Genotyping Extra de INNOGENETICS, que está basado en el principio de hibridación reversa. Parte de la región L1 del genoma de VPH se amplificó y los amplicones biotinilados desnaturalizados se hibridaron con sondas de oligonucleótidos específicas, las cuales se inmovilizaron en tiras de membranas que contenían 28 secuencias específicas de VPH. Este sistema detecta los genotipos de bajo riesgo oncogénico: 6, 11, 40, 43, 44, 54 y 70 ; los tipos de alto riesgo oncogénico: 16, 18, $31,33,35,39,45,51,52,56,58,59,68,73$ y 82. Así mismo, genotipos probables de alto riesgo, tales como: 26, 53, 66, 69/71 y 74. Después de la hibridación y el lavado astringente, la estreptavidina conjugada a fosfatasa alcalina se agregó a los híbridos biotinilados formados previamente. La incubación con el cromógeno BCIP/ NBT produjo un precipitado púrpura y los resultados se pudieron interpretar visualmente.

La PCR se llevó a cabo en un volumen final de $50 \mu \mathrm{L}$. Para ello $10 \mu \mathrm{L}$ del ADN extraído se colocó en la mezcla de reacción con 37,7 $\mu \mathrm{L}$ de AMP MIX y 2,3 $\mu \mathrm{L}$ de ENZ MIX. Las condiciones de corrida para la amplificación del ADN viral fueron las siguientes: 1 ciclo de descontaminación a $37^{\circ} \mathrm{C}$ por 10 minutos, 1 ciclo de desnaturalización inicial a $94^{\circ} \mathrm{C}$ por 9 minutos, seguido de 40 ciclos de desnaturalización a $94{ }^{\circ} \mathrm{C}$ por 30 segundos, hibridación a $52^{\circ} \mathrm{C}$ por 45 segundos, extensión a $72{ }^{\circ} \mathrm{C}$ por 45 segundos y una etapa de extensión final a $72^{\circ} \mathrm{C}$ por 5 minutos.

Interpretación de resultados. Todas las bandas claramente visibles se clasificaron utilizando la tarjeta de lectura de INNO-LiPA HPV Genotyping Extra. Los patrones de bandas se compararon con el diagrama de interpretación de INNO-LiPA HPV Genotyping Extra suministrado por el kit, el cual muestra las bandas positivas (filas) para los distintos tipos de VPH. Aquellas muestras para las que el patrón de bandas obtenido no pudo asignarse a ningún patrón de genotipo, o aquellas que no tenían bandas específicas de tipo (1-28), pero tenían como mínimo una banda de control positivo de $\mathrm{VPH}$, se clasificaron como VPH positivas, pero de tipo no identificable (VPHX).

Análisis estadístico. Para evaluar la posible relación de las variables en este estudio, se utilizó la prueba de chi-cuadrado $\left(\mathrm{X}^{2}\right)$, con el programa estadístico SPSS V.2.0. La significancia estadística fue considerada para valores de $\mathrm{p}<0,05$.

\section{Resultados}

Características demográficas de la muestra de estudio. La muestra de estudio estuvo constituida por 31 hisopados bucales de pacientes mayores de edad, VIH positivos, en el período de enero a marzo 2017. El promedio de edad de la muestra de estudio fue de 49,03 $\pm 11,48$ (rango: 25 - 75 años). El 74,19\% (23/31) de la muestra en este estudio, correspondió a hombres en edades comprendidas entre 26 y 75 años (promedio de edad: 48,65 $\pm 11,09$ años). El 25,81\% (8/31) correspondió a mujeres en edades comprendidas entre 25 a 67 años (promedio de edad: 50,13 $\pm 13,27$ años).

En general, el 58\% (18/31) de los pacientes que participaron en este estudio no tenían hábito tabáquico y el $42 \%(13 / 31)$ de los individuos si lo tenían, de los cuales las mujeres representaron el 6,5\% (2/31) y los hombres el 35,5\% (11/31). En cuanto al consumo de alcohol, el 
$16 \%(5 / 31)$ de los pacientes consumían alcohol, de estos el 13\% (4/31) eran hombres y el 3\% (1/31) restante era una mujer.

Marcadores inmunitarios de la evolución de la enfermedad en la muestra de estudio. En relación a la evaluación del estado inmunitario referido al contaje de linfocitos T CD4 y la carga viral de los pacientes incluidos en el estudio, en la Tabla 1 se puede observar lo siguiente: El 68\% (21/31) de los pacientes presentaron valores promedios de los linfocitos T CD4 mayores o iguales a 500 $\mathrm{cel} / \mathrm{mm}^{3}$, de los cuales el 52\% (16/31) eran hombres y el $16 \%$ eran mujeres (5/31). Del 29\% (9/31) de los individuos que presentó valores comprendidos entre 200- 499 $\mathrm{cel} / \mathrm{mm}^{3}$, el $19 \%$ (6/31) correspondía a hombres y el 10\% (3/31) a mujeres. Solo un 3,23\% (1/31) correspondiente a un hombre que presentó valores $<200 \mathrm{cel} / \mathrm{mm}^{3}$ (Tabla 1). Con respecto a la carga viral, el $42 \%$ (13/31) de los pacientes presentaban valores de carga viral $<20$ copias ARN/mL, de los cuales 36\% (11/31) eran hombres y el $6 \%(2 / 31)$ eran mujeres. Del 29\% (9/31) de la muestra de estudio que presentaban valores $<50$ copias ARN/mL, el 19\% (6/31) correspondió a hombres y el 10\% (3/31) a mujeres. Por último, de igual manera el 29\% (9/31) presentaban valores $>50$ copias $\mathrm{ARN} / \mathrm{mL}$, de los cuales el $19 \%(6 / 31)$ eran hombres y el 10\% (3/31) eran mujeres (Tabla 1). Es importante mencionar, que para el momento de la toma de muestra de hisopados bucales, todos los individuos (100\%) que participaron en este estudio se encontraban bajo terapia antirretroviral.

Frecuencia de la infección por VPH en la muestra de estudio. Para la detección de la presencia de VPH se empleó el sistema de hibridación reversa de INNO-LiPA, que detecta simultáneamente 28 genotipos de $\mathrm{VPH}$ y se encontró que el 61\% (19/31) de los pacientes presentaba una infección por VPH, mientras que el 39\% (12/31) no presentó infección por este virus. Es importante mencionar, que del total de pacientes positivos para VPH el 63,20\% (12/19) correspondía a hombres y el 36,80\% (7/19) eran mujeres.

Frecuencia de Genotipos de VPH presentes en la muestra de estudio. En general, en la muestra de estudio se observó la presencia de genotipos de VPH de alto y bajo riesgo oncogénico. Los genotipos de VPH-BR estuvieron presentes en el 84,20\% (16/19) de las muestras de estudio y los genotipos de VPH-AR en el 73,70\% (14/19). El genotipo 6 de bajo riesgo oncogénico fue el más prevalente con 73,70\% (14/19), el VPH-18 de alto riesgo oncogénico estuvo presente en el 63,20\% (12/19), el VPH-11 de bajo riesgo oncogénico se detectó en el 37,0\% (7/19), el VPH-16 de alto riesgo oncogénico se encontró en el 32\% (6/19), el VPH-35 de alto riesgo oncogénico se observó en el 5,30\% (1/19) y un paciente positivo para VPH, que no se pudo tipificar por la metodología empleada $(5,30 \%)$ (Figura 1$)$.

Tabla 1. Marcadores Inmunitarios de la muestra de estudio

\begin{tabular}{lccc}
\hline \multicolumn{1}{c}{ Marcadores estado inmunitario } & Masculino & Femenino & Total \\
\hline Contaje T CD4 cél/mm $\mathrm{mm}^{3}$ & & & $68 \%(21 / 31)$ \\
$>500$ & $52 \%(16 / 31)$ & $16 \%(5 / 31)$ & $29 \%(9 / 31)$ \\
$200-499$ & $19 \%(6 / 31)$ & $10 \%(3 / 31)$ & $3,23 \%(1 / 31)$ \\
$<200$ & $3,23 \%(1 / 31)$ & 0 & $42 \%(13 / 31)$ \\
\hline Carga Viral & & & $29 \%(9 / 31)$ \\
$<20$ copias ARN $/ \mathrm{mL}$ & $36 \%(11 / 31)$ & $6 \%(2 / 31)$ & $29 \%(9 / 31)$ \\
$<50$ copias ARN/mL & $19 \%(6 / 31)$ & $10 \%(3 / 31)$ & $10 \%(3 / 31)$ \\
$>50$ copias ARN $/ \mathrm{mL}$ & $19 \%(6 / 31)$ & & \\
\hline
\end{tabular}

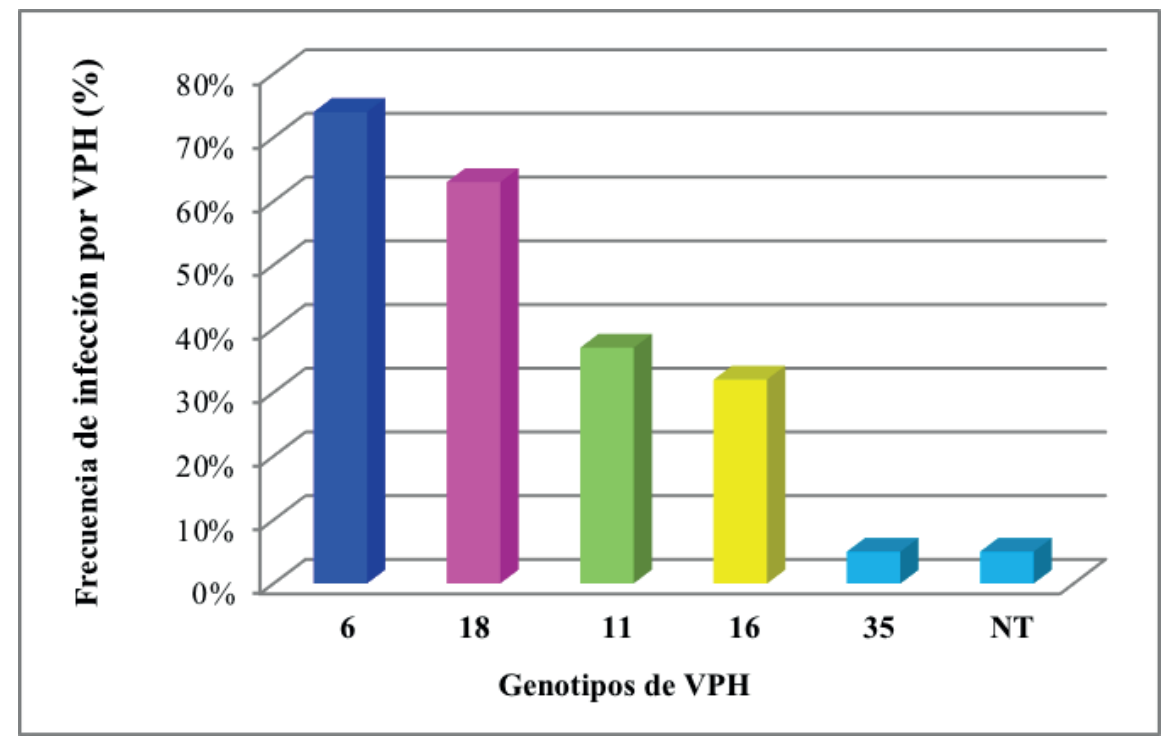

Figura 1. Frecuencia de los genotipos de VPH presentes en la muestra de estudio 
Es importante mencionar, que un 74\% (14/19) de los pacientes presentaron infecciones múltiples, es decir con más de un genotipo de VPH y un 26\% (5/19) que presentó infección con un solo genotipo de VPH. Entre las infecciones múltiples, la más frecuente fue la coinfección mixta con los genotipos de VPH-6/18 de bajo y alto riesgo oncogénico con $35,70 \%(5 / 14)$, seguida de un $21,40 \%(3 / 14)$ con los genotipos 6/11/18 e igualmente un $21,40 \%(3 / 14)$ con los genotipos 6/11, ambos de bajo riesgo oncogénico. Así mismo se detectó un
$14,30 \%(2 / 14)$ de coinfección mixta con los genotipos 6/11/16/18 de bajo y alto riesgo oncogénico y por último un $7,10 \%(1 / 14)$ de coinfección con los genotipos 11/16 de bajo y alto riesgo oncogénico (Figura 2). La Figura 3 muestra la detección y tipificación de algunas muestras mediante el Sistema de Hibridación Reversa de INNO-LiPA.

Frecuencia de pacientes con y sin infección por VPH con respecto al contaje de T CD4 (cél/ $\mathrm{mm}^{3}$ de sangre).

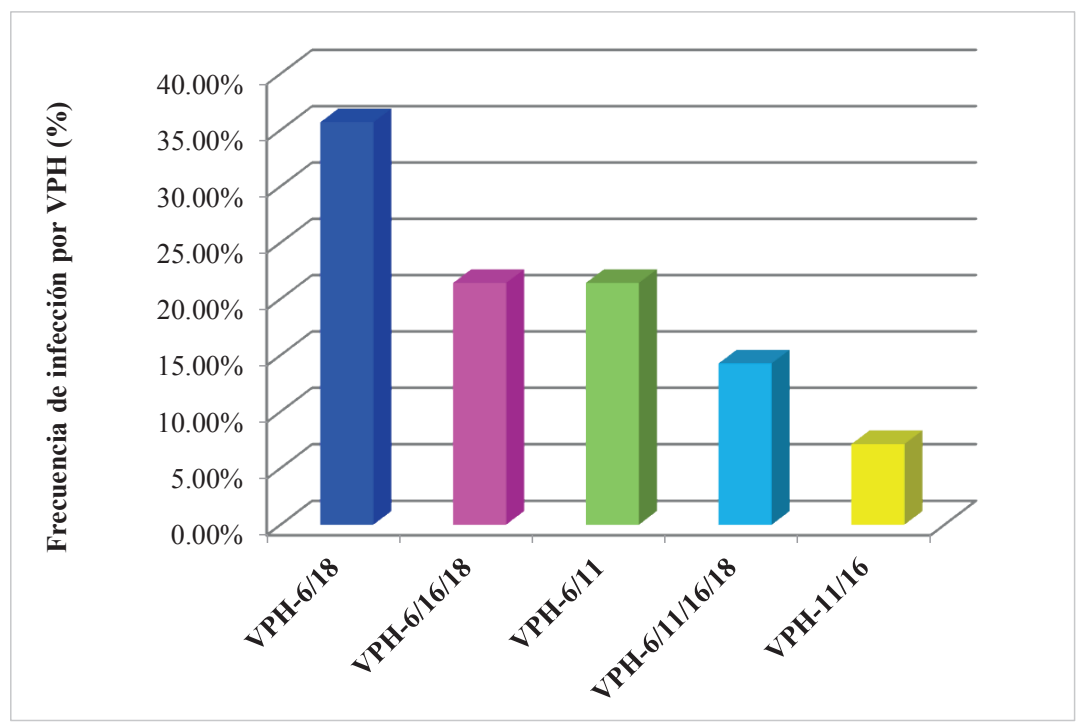

Figura 2. Frecuencia de infecciones múltiples de VPH presentes en la muestra de estudio

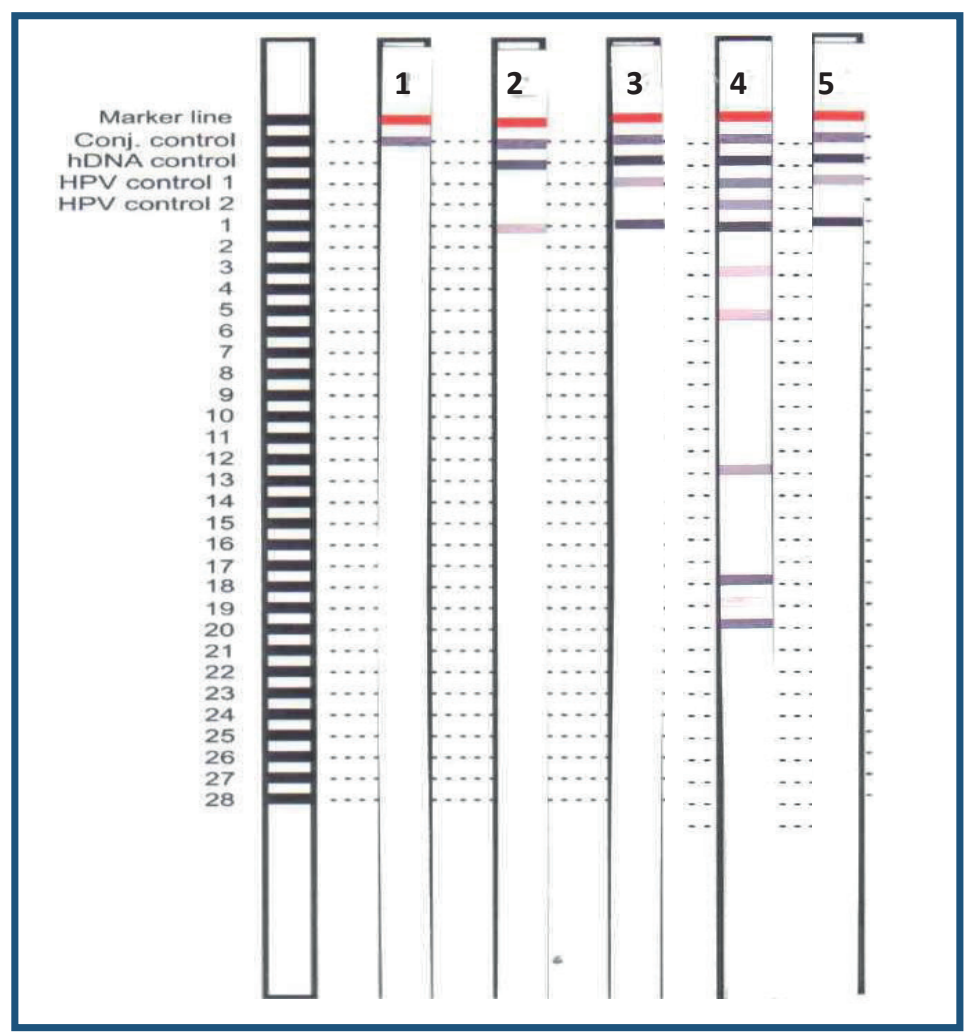

Figura 3. Detección y tipificación de VPH mediante el Sistema de Hibridación Reversa de INNO-LiPA. Línea 1: Control Negativo; Líneas 2 y 3: Muestras Positivas para VPH6; Línea 4: Muestra Positiva para VPH- 6, 16 y 18. Línea 5: Control Positivo 
$\mathrm{Al}$ evaluar la frecuencia de pacientes con y sin infección por VPH con respecto al contaje de linfocitos T CD4, se observó que el 57,90\% (11/19) de los individuos con infección por VPH y el 83,30\% (10/12) de los pacientes sin infección por este virus presentaban un contaje de linfocitos $>500$ (cél $/ \mathrm{mm}^{3}$ sangre) $(\mathrm{p}>0,140)$. Contrariamente, el 36,80\% (7/19) de los individuos con infección por $\mathrm{VPH}$ y el $16,70 \%(2 / 12)$ de los que no presentaban infección tenían recuentos de linfocitos entre 200 - 499 (cél $/ \mathrm{mm}^{3}$ sangre) $(\mathrm{p}>0,228)$. Solo un paciente positivo para VPH $(5,30 \%)$ presentó un recuento de linfocitos T CD $4<200$ (cél $/ \mathrm{mm}^{3}$ sangre) $(\mathrm{p}>0,419)$. En general, en este estudio la mayor frecuencia de pacientes negativos para la infección por VPH presentó un recuento de linfocitos $>500$ (cél $/ \mathrm{mm}^{3}$ sangre) al igual que para los individuos con infección por VPH (Tabla 2).

Frecuencia de pacientes con y sin infección por VPH con respecto a la carga viral. Con respecto a la frecuencia de pacientes con y sin infección por el VPH en relación a la carga viral, se observó que no hubo correlación entre la carga viral y la presencia de la infección por VPH ( $>0,05)$. Los resultados muestran que el 50\% (6/12) de los pacientes VPH negativos presentaban una carga viral $<20$ copias $/ \mathrm{mL}$, seguido de un $25 \%(3 / 12)$ que tenían un valor de carga viral $<50$ copias $/ \mathrm{mL}$ y un $16,66 \%(2 / 12)$ con valores de carga viral entre 51- 20 000 copias $/ \mathrm{mL}$. Se observó un solo paciente VPH negativo con un valor de carga viral $>20000$ copias $/ \mathrm{mL}$. Igualmente, el 42,10\% (8/19) de los pacientes VPH positivos tenían una carga viral $<20$ copias $/ \mathrm{mL}$, seguido de un $31,60 \%(6 / 19)$ que presentaban valores de carga viral $<50$ copias $/ \mathrm{mL}$ y por último, un $26,30 \%(5 / 19)$ de pacientes VPH positivos con un valor de carga viral entre 51- 20000 copias/mL (Tabla 3).

\section{Discusión}

Las manifestaciones bucales de la infección por VIH forman una parte importante de la enfermedad desde las primeras descripciones y son componentes indicativos de su progresión. En algún momento de la infección, en nueve de cada diez pacientes, van a aparecer manifestaciones bucales, y en algunas ocasiones estas manifestaciones van a ser el primer signo de la enfermedad, por lo tanto tienen un importante valor diagnóstico y predictivo ${ }^{25}$

Se han registrado más de 40 manifestaciones bucales asociadas en mayor o menor medida con la infección por VIH y se han agrupado según su origen en micóticas, bacterianas y víricas. Sin embargo, es importante mencionar que desde la introducción de la HAART se ha observado una disminución significativa de las manifestaciones bucales cercana al 30\% asociadas a la inmunodeficiencia, tanto en su frecuencia como en su gravedad tales como: leucoplasia vellosa bucal y la periodontitis ulcerativa necrotizante o gingivitis ulceronecrotizante aguda, entre otras, sin observarse una reducción en la incidencia de la candidiasis oral, ulceras bucales o del sarcoma de Kaposi ${ }^{2,3}$

Así mismo, se ha reportado que el grado de reconstitución inmunitaria alcanzado con esta terapia controla otras infecciones oportunistas asociadas con SIDA y causadas por virus, como: el virus herpes simple (HSV), el citomegalovirus (CMV), y el virus Epstein Barr (VEB), entre otros ${ }^{26}$. En contraste, otros estudios muestran un marcado incremento de la incidencia de verrugas orales, condilomas e hiperplasia epitelial multifocal producidas por VPH, en pacientes tratados con HAART 14,15, 27,28 .

Una investigación realizada en el año 2013, donde se demostró que las proteínas tat y gp120 del VIH alteran las uniones estrechas en el epitelio de la mucosa bucal, un factor que puede facilitar la infección tras la exposición ${ }^{29}$, puede explicar el incremento en la incidencia de lesiones asociadas a la infección por VPH en pacientes VIH positivos. Así mismo, se ha reportado que la inmunosupresión local y/ o sistémica puede promover la reactivación de una infección latente previamente adquirida y/ o la adquisición de nuevas infecciones por VPH en

Tabla 2. Frecuencia de pacientes con o sin infección por VPH con respecto al contaje de CD4 (cél/mm³ de sangre)

\begin{tabular}{|c|c|c|c|c|c|}
\hline \multirow[b]{2}{*}{$\begin{array}{c}\text { Contaje de CD4 } \\
\left(\text { cél } / \mathrm{mm}^{3}\right)\end{array}$} & \multicolumn{2}{|c|}{ Pacientes sin infección } & \multicolumn{2}{|c|}{ Pacientes con infección } & \multirow[b]{2}{*}{$\begin{array}{c}\text { Prueba }\left(\mathrm{x}^{2}\right) \\
\text { Valor } \mathrm{p}\end{array}$} \\
\hline & $\begin{array}{c}\text { Promedio } \\
\text { CD4 }\end{array}$ & $\%$ VPH- & $\begin{array}{l}\text { Promedio } \\
\text { CD4 }\end{array}$ & $\% \mathrm{VPH}+$ & \\
\hline$>500$ & 737,3 & $83,30 \%(10 / 12)$ & 682,72 & $57,90 \%(11 / 19)$ & 0,140 \\
\hline 200- 499 & 296,5 & $16,70 \%(2 / 12)$ & 432,14 & $36,80 \%(7 / 19)$ & 0,228 \\
\hline$<200$ & 0 & 0 & 0 & $5,30 \%(1 / 19)$ & 0,419 \\
\hline
\end{tabular}

Tabla 3. Frecuencia de pacientes con y sin infección por VPH con respecto a la carga viral (copias ARN viral/mL)

\begin{tabular}{ccc}
\hline $\begin{array}{c}\text { Carga Viral } \\
\text { (copias ARN viral/mL) }\end{array}$ & VPH+ & VPH- \\
\hline$<\mathbf{2 0}$ & $42,10 \%(8 / 19)$ & $50 \%(6 / 12)$ \\
\hline$<\mathbf{5 0}$ & $31,60 \%(6 / 19)$ & $25 \%(3 / 12)$ \\
\hline $\mathbf{5 1 - 2 0 0 0 0}$ & $26,30 \%(5 / 19)$ & $16,70 \%(2 / 12)$ \\
\hline$>\mathbf{2 0 0 0 0}$ & 0 & $8,30 \%(1 / 12)$ \\
\hline
\end{tabular}


la mucosa bucal ${ }^{30}$, es así como en los últimos años ha cobrado especial interés la identificación de infecciones virales y particularmente de los VPH en la mucosa bucal de pacientes VIH positivos.

En particular, este trabajo de investigación tuvo como objetivo general, establecer la prevalencia de la infección por VPH en la cavidad bucal de un grupo de individuos VIH positivos bajo terapia antirretroviral y correlacionarlo con el estado inmunitario: contaje de linfocitos $\mathrm{T}$ CD4 y carga viral de VIH. En este estudio, se observó que el $61 \%$ de los pacientes presentaban infección por $\mathrm{VPH}$, mientras que el 39\% no presentaba infección con este virus. Sin embargo, otras investigaciones han reportado prevalencias variables de la infección por VPH en la cavidad bucal de pacientes VIH positivos que van desde un $14 \%$ hasta un $52 \%$, esta diferencia puede atribuirse a la metodología empleada para la detección del virus y el tipo de muestra utilizada ${ }^{10-12,31,32}$. Es importante mencionar, que esta variabilidad en la detección de la infección por VPH en la cavidad bucal, ha hecho difícil realizar estudios comparativos y analizar los datos existentes de distintas investigaciones acerca de la prevalencia de estos virus en la mucosa bucal normal, así como estudiar la asociación de la infección por VPH y el cáncer bucal ${ }^{33}$.

La alta prevalencia de la infección por VPH observada en nuestro estudio, puede ser explicada por el empleo de una metodología altamente sensible y específica como es el Sistema de Hibridación Reversa de INNO-LiPA, que combina la amplificación de un fragmento pequeño de 65 pb y la hibridación con sondas de ADN, capaces de detectar hasta 28 genotipos diferentes, a diferencia de los métodos convencionales que utilizan los oligonucleótidos MY09/MY11 correspondientes a una región altamente conservada del gen $L 1$, que amplifican un fragmento de mayor tamaño de 450 pb. Sin embargo, en un estudio realizado también en muestras bucales de individuos VIH positivos, en edades comprendidas entre 50- 69 años de edad, donde se utilizó un sistema de PCR optimizado para la detección de VPH cutáneos y de mucosa, que empleó una combinación de oligonucleótidos MY09/MY11 y oligonucleótidos degenerados FAP59/64 correspondientes también a la región $L 1$ del virus, reportó una infección por VPH de 87\%, más alta que la reportada en nuestro estudio debido a la sensibilidad del método ${ }^{34}$.

Así mismo, la alta prevalencia en nuestro estudio además puede ser explicada porque todos los individuos que participaron en esta investigación, se encontraban bajo terapia antirretroviral. Diferentes investigaciones de manera consistente han reportado que desde la introducción de la terapia antirretroviral altamente activa en pacientes con VIH ha ocurrido una reducción importante en las tasas de morbilidad y mortalidad, y los pacientes viven más tiempo con una mejor calidad de vida, sin embargo, junto con el aumento de la esperanza de vida, la incidencia de comorbilidades y en particular la infección por VPH también ha aumentado ${ }^{35-37}$.
En este sentido, diferentes grupos de investigación han demostrado que los pacientes VIH positivos tienen mayor riesgo de desarrollar cánceres asociados a la infección por los VPH de alto riesgo oncogénico, incluyendo los cánceres cervicales, de ano y orofaringe ${ }^{38-40}$. Sin embargo, la incidencia para estos cánceres entre los individuos VIH positivos comparado a la población general varía considerablemente dependiendo del sitio anatómico. Por ejemplo, los individuos VIH positivos tienen 25 veces más riesgo de desarrollar cáncer anal con respecto a la población general y en el caso del cáncer de orofaringe, tienen entre 2 a 6 veces mayor riesgo para desarrollar esta patología ${ }^{41,42}$.

Un estudio realizado en el año 2015, acerca de la historia natural de la infección por VPH en la mucosa bucal entre individuos con y sin infección por el VIH, se observó que el comportamiento sexual, la infección por VIH y la gravedad de la inmunosupresión aumentaban significativamente el riesgo de infección de la mucosa bucal por VPH. En contraste, factores como la edad avanzada, sexo masculino y hábito tabáquico incrementaban el riesgo de persistencia ${ }^{43}$. Sin embargo, es importante mencionar que al igual que en las infecciones anogenitales, las infecciones bucales por VPH parecen ser a menudo transitorias, a pesar de que algunas de estas infecciones pueden persistir hasta 2 años ${ }^{44-46}$. Hasta el momento, se desconoce el riesgo de progresión de estas infecciones persistentes por largo tiempo en el desarrollo del cáncer orofaringeo, por lo tanto se requieren más investigaciones para comprender mejor la persistencia de la infección por estos virus, así como las bases biológicas de las asociaciones con el sexo, la edad y el tabaquismo ${ }^{43}$.

En relación a los genotipos de $\mathrm{VPH}$, diversos estudios han mostrado que las personas infectadas con el VIH son más propensas a estar infectadas con genotipos de alto riesgo oncogénico en la mucosa bucal ${ }^{47-49}$. En vista del aumento en la edad de los pacientes con HAART a largo plazo y el posible papel etiológico del VPH en la carcinogénesis bucal, estos individuos coinfectados por VIH/VPH pueden estar en riesgo significativo de desarrollar lesiones y neoplasias malignas asociadas a la infección por VPH ${ }^{50,51}$.

En general, en este estudio se observó que el 84,20\% de los individuos presentaban infección con VPH de bajo riesgo oncogénico y los $\mathrm{VPH}$ de alto riesgo oncogénico estuvieron presentes en el 73,70\% de los individuos. En cuanto a los genotipos de VPH de bajo riesgo oncogénico, el genotipo 6 fue el más prevalente con 73,68\%, seguido del tipo 11 que se detectó en el $37 \%$ de los individuos, ambos genotipos asociados con el desarrollo de displasias menores, como los papilomas escamosos. Con respecto a los genotipos de VPH de alto riesgo oncogénico el tipo 18 estuvo presente en el 63,16\%, seguido del VPH tipo 16 en el 32\%. La prevalencia en nuestro estudio de los genotipos 16 y 18 causantes de aproximadamente el $70 \%$ de los cánceres de cuello uterino a nivel mundial, fueron significativamente mayores que las reportadas en otra investigación, donde se observó una prevalencia del $6 \%$ y del $2 \%$ de los genotipos de 
VPH- 16 y 18, respectivamente ${ }^{52}$. El VPH tipo 35 considerado también de alto riesgo oncogénico, se observó en el 5,26\% y solo en un paciente positivo para VPH, no se puedo identificar el genotipo por la metodología empleada $(5,26 \%)$.

Un hallazgo importante en esta investigación, fue la detección de infecciones con múltiples genotipos de VPH en el $74 \%$ de los pacientes, lo que coincide con los resultados obtenidos en otra investigación, donde realizaron la detección de VPH en muestras bucales de pacientes VIH positivos y reportaron hasta un $71 \%$ de infecciones múltiples por $\mathrm{VPH}$, mientras que solo un $26 \%$ de los individuos presentaron infección con un solo genotipo de $\mathrm{VPH}^{52}$. Estos resultados son diferentes a lo reportado en otro estudio, que mostró que solo el $8 \%$ de los individuos VIH positivos presentaban infección en la mucosa bucal con un solo genotipo de VPH ${ }^{34}$.

Con respecto a la frecuencia de la infección por VPH en relación al contaje de linfocitos T CD4, en este estudio, solo un paciente tenía un contaje de $<200$ cél $/ \mathrm{mm}^{3}$ consistente con SIDA, lo que puede explicar la falta de significancia estadística $(p>0,05)$ entre la detección de $\mathrm{VPH}$ en la mucosa bucal y el estado inmunitario. Así mismo en relación a la carga viral, solo un paciente tenía valores >10 000 copias/mL, lo que también puede explicar la falta de significancia estadística entre la presencia de la infección y la carga viral de VIH, posiblemente debido a que todos los pacientes que participaron en este estudio se encontraban bajo terapia antirretroviral, lo que es consistente con diversos trabajos, que han demostrado que entre los factores que facilitan la aparición de manifestaciones bucales se encuentran: un recuento de linfocitos T CD4 menor de 200 cél $/ \mathrm{mm}^{3}$ y una carga viral mayor de 10000 copias $/ \mathrm{mL}$, además de otros factores locales como la xerostomía, una mala higiene bucal y el consumo de tabaco entre otros que también intervienen en la patología bucal ${ }^{2,3}$.

En este estudio, la alta frecuencia de infección por VPHAR y de infecciones con múltiples genotipos observada en la cavidad bucal de individuos VIH positivos, que no presentaron lesiones compatibles con esta infección al momento del examen extra e intrabucal, indica que los métodos moleculares de diagnóstico son de gran importancia en la detección de la infección subclínica y latente por estos virus. Así mismo, permite un seguimiento y manejo más oportuno de estos pacientes con mayor riesgo de desarrollar neoplasias malignas en la cavidad bucal.

El amplio espectro de genotipos de VPH detectados e infecciones múltiples, sugiere que la cavidad bucal en el contexto de la inmunosupresión puede ser un reservorio de infección subclinica, que pudiera tener importantes implicaciones en el paciente coinfectado VIH/ VPH, cuya esperanza de vida ha mejorado dramáticamente desde la introducción de la HAART.

\section{Referencias bibliográficas}

1. Avendaño L. Retrovirus. En Avendaño L., Ferrés M., Spencer E. Virología Clínica. Santiago de Chile: Mediterráneo; 2012. p. 241-256.
2. Grando L, Yurgel L, Machado D, Nachman S, Ferguson $\mathrm{F}$, Berentsen B, et al. The association between oral manifestations and the socioeconomic and cultural characteristics of HIV-infected children in Brazil and in the United States of America. Rev Panam Salud Publica. 2003;14:112-8.

3. Ceballos A, Gaitán L, Ceballos L, Lezama D. Oral lesions in HIV/AIDS patients undergoing highly active antiretroviral treatment including protease inhibitors: A new face of oral AIDS? AIDS Patient Care STDS. 2000;12:627- 35 .

4. Abbas A. Inmunodeficiencias congénitas y adquiridas. En Abbas A., Lichtman A., Pillai S. Inmunología Celular y Molecular. 6ta. Ed. Barcelona: Elsevier España; 2008. p. $463-488$.

5. Diaz-Dios P, Ocampo A., Miralles C. Changing prevalence of human immunodeficiency virus-associated oral lesions. Oral Surg Oral Med Oral Pathol Oral Radiol Endod. 2000;90(4):403-4.

6. Nokta M. Oral manifestations associated with HIV infection. Curr HIV/AIDS Rep. 2008;5(1):5-12.

7. Shiboski C. HIV-related oral disease epidemiology among women: year 2000 update. Oral Dis. 2002; 8:44-48.

8. Cherry-Peppers G, Daniels C, Meeks V, Sanders C, Reznik D. Oral manifestations in the era of HAART. J Natl Med Assoc. 2003;95:21-32.

9. Delgado A, Vallejo E, Marlos E. Oral manifestations of HIV infection in infants: a review article. Med Oral Patol Oral Cir Bucal. 2004;9:410-420.

10. Katz M, Greenspan D, Westenhouse J. Progression to AIDS in HIV infected homosexual men with, hairy leukoplakia and oral candidacies. AIDS. 1992; 6:95-100.

11. Feigal D, Katz M, Greenspan D. The prevalence of oral lesions in infected homosexual and bisexual men: Three San Francisco epidemiological cohorts. AIDS. 1991; 5:519-525.

12. Tappuni A, Flemming G. The effect of antiretroviral therapy on the prevalence of oral manifestations in HIV-infected patients: a UK study. Oral Surg Oral Med Oral Pathol Oral Radiol Endod. 2001;92(6):623-8.

13. Ramírez-Amador V, Esquivel-Pedraza L, Sierra-Madero J, Anaya-Saavedra G, González-Ramírez I, Ponce de León $\mathrm{S}$. The changing clinical spectrum of human immunodeficiency virus (HIV)-related oral lesions in 1,000 consecutive patients. A twelve-year study in a referral center in Mexico. Medicine. 2003;82:39-50.

14. King M, Reznik D, O’Daniels C, Larsen N, Osterholt D., Blumberg H. Human Papillomavirus-Associated Oral Warts among HIV-Seropositive Patients in the Era of Highly Active Antiretroviral Therapy: An Emerging Infection. Clin Infect Dis. 2002;34:641-8.

15. Schmidt-Westhausen A, Priepke F, Bergmann F, Reichart P. Decline in the rate of oral opportunistic infections following introduction of highly active antiretroviral therapy. J Oral Pathol Med. 2000;29:336-41.

16. Munoz N. Human papillomavirus and cancer: the epidemiological evidence. J Clin Virol. 2000;19:1- 5. 
17. Burd E. Human Papillomavirus and Cervical Cancer. Clin Microbiol Rev. 2003;16:1-17.

18. Kumar A, Pandya S, Mehrotra R, Bharti A, Singh M, Singh M. Comparative study between the Hybrid Capture II test and PCR based assay for the detection of human papillomavirus DNA in oral submucous fibrosis and oral squamous cell carcinoma. J Virol. 2010;7:253.

19. Martínez A, Baldiris R, Díaz A. Papiloma bucal producido por VPH y su relación con carcinoma. Rev Clín Med Fam. 2012;5(2):144-145.

20. D'Souza G, Kreimer A, Viscidi R, Pawlita M, Fakhry C, Koch W, et al. Case-control study of human papillomavirus and oropharyngeal cancer. $\mathrm{N}$ Engl J Med. 2007;356(19):1944-1956.

21. Cameron J, Mercante D, O’brien M, Gaffga A, Leigh J, Fidel P, Hagensee M. The Impact of Highly Active Antiretroviral Therapy and Immunodeficiency on Human Papillomavirus Infection of the Oral Cavity of Human Immunodeficiency Virus-Seropositive Adults. Sex Trans Dis. 2005;32(11):703- 709 .

22. Kreimer A, Alberg A, Daniel R, Gravitt P, Viscidi R, Garrett $\mathrm{E}$, et al. Oral human papillomavirus infection in adults is associated with sexual behavior and HIV serostatus. J Infect Dis. 2004;189:686- 698.

23. Mooij S, Boot H, Speksnijder A, Stolte I, Meijer C, et al. Oral human papillomavirus infection in HIV-negative and HIV-infected men who have sex with men: the HIV \& HPV in MSM (H2M) study. AIDS. 2013;27:21172128.

24. Fatahzadeh M, Schlecht N, Chen Z, Bottalico D, McKinney S, Ostoloza J, et al. Oral Human Papillomavirus Detection in Older Adults Who Have Human Immunodeficiency Virus Infection. Oral Surg Oral Med Oral Pathol Oral Radiol. 2013;115(4):505- 514.

25. Birnbaum W, Hodgson T, Reichart P, Sherson W, Nittayannanta S, Axell T. Prognostic significance of HIV-associated oral lesions and their relation to therapy. Oral Dis. 2002;8:110-4.

26. Buchacz K, Baker R, Palella F, Chmiel J, Lichtenstein $\mathrm{K}$, Novak R, et al. AIDS-defining opportunistic illnesses in US patients, 1994-2007: a cohort study. AIDS. 2010;24:1549-1559.

27. Eyeson J, Tenant-Flowers M, Cooper D, Johnson N, Warnakulasuriya K. Oral manifestations of an HIV positive cohort in the era of highly active anti-retroviral therapy (HAART) in South London. J Oral Pathol Med. 2002;31:169- 74 .

28. Challacombe S, Coogan M, Williams D. Overview of the Fourth International Workshop on the Oral Manifestations of HIV Infection. Oral Dis. 2002;8:9- 14

29. Tugizov S, Herrera R, Chin-Hong P, Veluppillai P, Greenspan D, Michael B, et al. HIV-associated disruption of mucosal epithelium facilitates paracellular penetration by human papillomavirus. Virology. 2013;446(12):378- 388 .

30. Safaeian M, Porras C, Schiffman M. Epidemiological study of anti-HPV16/18 seropositivity and subsequent risk of HPV16 and -18 infections. J Natl Cancer Inst. 2010;102(21):1653- 1662 .
31. Greenspan D, Greenspan J, Pindborg J, Schiodt M. El SIDA en la cavidad bucal. 1era. Edic. en Español. Caracas. 1994. Actualidades Médico Odontológicas Latinoamericanas.

32. Migliorati C, Migliorati E. Oral lesions and HIV. An approach to the diagnosis of oral mucosal lesions for the dentist in private practice. Schweiz Monatsschz Zahnmed. 1997;107:860-71.

33. Patil N, Chaurasia V, Babaji P, Ramesh D, Jhamb K., Sharma A. The effect of highly active antiretroviral therapy on the prevalence of oral manifestation in human immunodeficiency virus-infected patients in Karnataka, India. Eur J Dent. 2015;9:47-52.

34. Fatahzadeh M, Schlecht N, Chen Z, Bottalico D, McKinney S, Ostoloza J, et al. Oral Human Papillomavirus Detection in Older Adults Who Have Human Immunodeficiency Virus Infection. Oral Surg Oral Med Oral Pathol Oral Radiol. 2013;115(4):505- 514.

35. Ponnam S, Srivastava G, Theruru K. Oral manifestations of human immunodeficiency virus in children: An institutional study at highly active antiretroviral therapy centre in India. J Oral Maxillofac Pathol. 2012;16:195- 202.

36. Brady M, Oleske J, Williams P, Elgie C, Mofenson L, Dankner W. et al. Declines in mortality rates and changes in causes of death in HIV-1-infected children during the HAART era. J Acquir Immune Defic Syndr. 2010;53:86-94.

37. Konopnicki D, Mocroft A, de Wit S, Antunes F, Ledergerber B, Katlama C, et al. Hepatitis B and HIV: Prevalence, AIDS progression, response to highly active antiretroviral therapy and increased mortality in the EuroSIDA cohort. AIDS. 2005;19:593- 601.

38. Gillison ML, Broutian T, Pickard R, Tong Z, Xiao W, et al. Prevalence of Oral HPV Infection in the United States, 2009-2010. JAMA. 2012;307:693-703.

39. Engels E, Biggar R, Hall H, Cross H, Crutchfield A, Finch J, et al. Cancer risk in people infected with human immunodeficiency virus in the United States. Int J Cancer. 2008;123:187- 194.

40. Shiels M, Pfeiffer R, Gail M, Hall H, Li J, Chaturvedi A, et al. Cancer burden in the HIV-infected population in the United States. J Natl Cancer Inst. 2011;103:753-62.

41. Shiels M, Cole S, Kirk G, Poole C. A meta-analysis of the incidence of non-AIDS cancers in HIV-infected individuals. J Acquir Immune Defic Syndr. 2009;52:611-22.

42. Dubrow R, Silverberg M, Park L, Crothers K, Justice A. HIV infection, aging, and immune function: implications for cancer risk and prevention. Curr Opin Oncol. 2012;24(5):506-16.

43. Beachler D, Sugar E, Margolick J, Weber K, Strickler H, Wiley D, et al. Risk Factors for Acquisition and Clearance of Oral Human Papillomavirus Infection Among HIV-Infected and HIV-Uninfected Adults. Am J Epidemiol. 2015;181(1):40-53.

44. De Pokomandy A, Rouleau D, Ghattas G, Vézina S, Coté P, Macleod J, et al. Prevalence, clearance, and incidence of anal human papillomavirus infection in HIV-infected men: the HIPVIRG cohort study. J Infect Dis. 2009;199(7):965- 973. 
45. Strickler H, Burk R, Fazzari M, Anastos K, Minkoff $\mathrm{H}$, Massad L, et al. Natural history and possible reactivation of human papillomavirus in human immunodeficiency virus-positive women. J Natl Cancer Inst. 2005;97(8):577-586.

46. Rositch A, Koshiol J, Hudgens M, Razzaghi H, Backes $\mathrm{D}$, Pimenta J, et al. Patterns of persistent genital human papillomavirus infection among women worldwide: a literature review and meta-analysis. Int J Cancer. 2012;131(6):1271-1285.

47. Kreimer A, Alberg A, Daniel R, Gravitt P, Viscidi R, Garrett $\mathrm{E}$, et al. Oral human papillomavirus infection in adults is associated with sexual behavior and HIV serostatus. J Infect Dis. 2004;189:686-698.

48. Fakhry C, D'souza G, Sugar E, Weber K, Goshu E, Minkoff $\mathrm{H}$, et al. Relationship between Prevalent Oral and Cervical Human Papillomavirus Infections in $\mathrm{Hu}-$ man mmunodeficiency Virus- Positive and -Negative Women. J Clin Microbiol. 2006;44:4479- 85.
49. Sireraa G, Videla S, Pinol M, Canadas M, Llatjos M, Ballesters A, et al. High prevalence of human papillomavirus infection in the anus, penis and mouth in HIV-positive men. AIDS. 2006;20:1201-4.

50. D'Souza G, Kreimer A, Viscidi R, Pawlita M, Fakhry C, Koch W, et al. Case-control study of human papillomavirus and oropharyngeal cancer. N Engl J Med. 2007;356(19):1944-1956.

51. Hagensee M, Cameron J, Leigh J, Clark R. Human papillomavirus infection and disease in HIV-infected individuals. Am J Med Sci. 2004;328:57- 63.

52. Beachler D., Weber K., Margolick J., Strickler H., Cranston R., et al. Risk factors for oral HPV infection among a high prevalence population of HIV-positive and at-risk HIV-negative adults. Cancer Epidemiol Biomarkers Prev. 2012:21: 122-133. 